\title{
Criteria for identification of Schistosoma mansoni eggs in faecal sediments prepared with the Helmintex method and stained by ninhydrin
}

\author{
Renata Perotto de Souza, Vivian Favero, Vanessa Fey Pascoal, Catieli Lindholz, \\ Hélio Radke Bittencourt, Carlos Graeff-Teixeira/ ${ }^{+}$
}

Pontifícia Universidade Católica do Rio Grande do Sul, Escola de Ciências, Porto Alegre, RS, Brasil

\begin{abstract}
Helmintex is a sensitive method used for detecting Schistosoma mansoni eggs. Here, we describe the observed frequency of six proposed criteria associated with the identification of $S$. mansoni eggs prepared with the Helmintex method and stained with ninhydrin. The efficacy of these criteria in classifying $S$. mansoni eggs when applied in various combinations was also examined. Nine observers registered the presence or absence of 6 different criteria in 100 eggs using a microscope at 100x magnification. Ninhydrin purple, which was frequently observed, was the criterion associated with the lowest inter-observer variability. At least three criteria were associated with a significantly better performance in egg identification. In conclusion, ninhydrin staining and a combination of criteria are recommended for microscope examination of faecal sediments.
\end{abstract}

Key words: schistosomiasis - egg-detection - ninhydrin - coproparasitology

An increasing number of countries have successfully implemented schistosomiasis control programs aimed at either eliminating this public health problem or interrupting its transmission. ${ }^{(1,2,3)}$ However, in areas with low infection intensity, classical diagnostic methods lack the sensitivity needed for diagnosing schistosomiasis. ${ }^{(4,5)} \mathrm{Hel}-$ mintex (HTX) method is a procedure which has been recently developed for diagnosing schistosomiasis. ${ }^{(6)}$ This method uses the interaction between Schistosoma eggs and paramagnetic particles in a magnetic field to obtain a product that can then be stained using ninhydrin. ${ }^{(7)}$ However, despite the high sensitivity of this method, it involves a laborious process even after several modifications, such as new sieving procedures, inclusion of detergent in the fixation solution, and staining of eggs with ninhydrin for microscopic examination. ${ }^{(8)}$ Although the HTX method is not a "point-of-care" diagnostic method, it is considered as a reference method for detecting schistosomiasis due to its extremely high sensitivity. ${ }^{(9)}$

Favero et al., ${ }^{(8)}$ proposed a set of criteria for the identification of $S$. mansoni (Sm) eggs in preparations obtained through the HTX method and stained with ninhydrin . These criteria include: 1) the presence of spines; 2) egg size: $\left.150-117 \mu \mathrm{m} \times 70-40 \mu \mathrm{m} ;{ }^{(10)} 3\right)$ egg shape; 4) definition of shell line; 5) internal space, and 6) colour. The objective of the current study was to evaluate these criteria by: (i) describing the frequency with which individual and combinatorial subsets of these six criteria occurred and (ii) evaluating the usefulness of these criteria for identifying $S$. mansoni eggs.

doi: 10.1590/0074-02760180529

Financial support: CG-T is a recipient of a CNPq PQ 1D fellowship and grants 307005/2014-3. RP-S is a recipient of a BPA-PUCRS fellowship.

+ Corresponding author: graeff.teixeira@gmail.com

(D) https://orcid.org/0000-0003-2725-0061

Received 8 November 2018

Accepted 24 April 2019
Filter papers containing positive faecal sediments were originally prepared as a part of a field study conducted in Candeal, Sergipe, in northeastern Brazil. The original study was approved by the Pontifícia Universidade Católica do Rio Grande do Sul (PUCRS) ethical committee (register 48809715.1.0000.5336) and the field and laboratory protocols used are described elsewhere. .9) $^{(9)}$ These filter papers were selected for re-examination in the present study.

The criteria for identifying $S$. mansoni eggs in HTX preparations stained with ninhydrin were proposed by experienced observers at the GPB-PUCRS laboratory. These six criteria were paired according to their contribution to a definitive diagnosis of Sm eggs (Fig. 1). The criteria were as follows: group A, criterion 1 - presence of lateral spine; criterion $2-$ a well-defined shell outline; group B, criterion 1 - ovoid shape; criterion 2 - size; group $C$, criterion 1 - space between shell and miracidium; criterion 2 - purple colour. Different combinations of criteria from groups A, B, and C were also analysed in the following subsets: combination $1-$ the use of criteria from only 1 group; combination $2-$ the use of criteria from any two groups; and combination 3 - having at least one criterion from all three groups.

A group of nine readers simultaneously observed selected fields containing a total of 100 eggs on a flat screen monitor, and recorded their observations regarding the six possible criteria on a standardised form. All images shown were visualised with a ZEISS AXIO microscope equipped with a QIMAGE RESTINGA 7 camera and IMAGE PRO 7 software. The projected images were obtained at 100x magnification. Graphs were generated and statistical analyses were performed via IBM SPSS 20. Frequencies and confidence intervals (CI) for proportions were computed to compare the prevalence of criteria.

The proportional frequencies (the frequencies relative to the total number of observations) of each observed criterion ranged from $58 \%$ (shape) to $99 \%$ (purple 
colour) (Table). The criteria "ovoid shape" and "well-defined shell outline" (Fig. 1), had the highest inter-observer frequency variability, with $95 \% \mathrm{CI}$ from $54.9 \%$ to $61.3 \%$ (range: 6.4 ) and $70.2 \%$ to $76 \%$ (range: 5.8 ), respectively. The best performance and highest inter-observer agreement were associated with combination 3 (observing at least one criterion from each group) (Fig. 2).

The classification of criteria into groups A, B, and $\mathrm{C}$ was based on decreasing specificity for identifying S. mansoni eggs from other helminth eggs (Fig. 1). The presence of a lateral spine is a unique characteristic of $S$. mansoni eggs, and the use of this criterion for detecting S. mansoni eggs constitutes a highly specific diagnostic method. The criterion, "well-defined shell outline", refers to a striking feature of many helminth eggs, such as eggs of Ancylostoma spp. and Enterobius spp., and the possibility that faecal sediment debris will present these features is very low. Undeformed eggs may present an appropriate size and/or shape for identification (e.g., group B criteria).

The purple colour produced by ninhydrin staining (group C criteria) is very helpful for quickly spotting eggs during initial microscopic screening of the filters. However, other debris may also be stained purple. While the purple colour criterion had the highest detection prevalence $(99.7 \%)$ and the lowest inter-observer var-

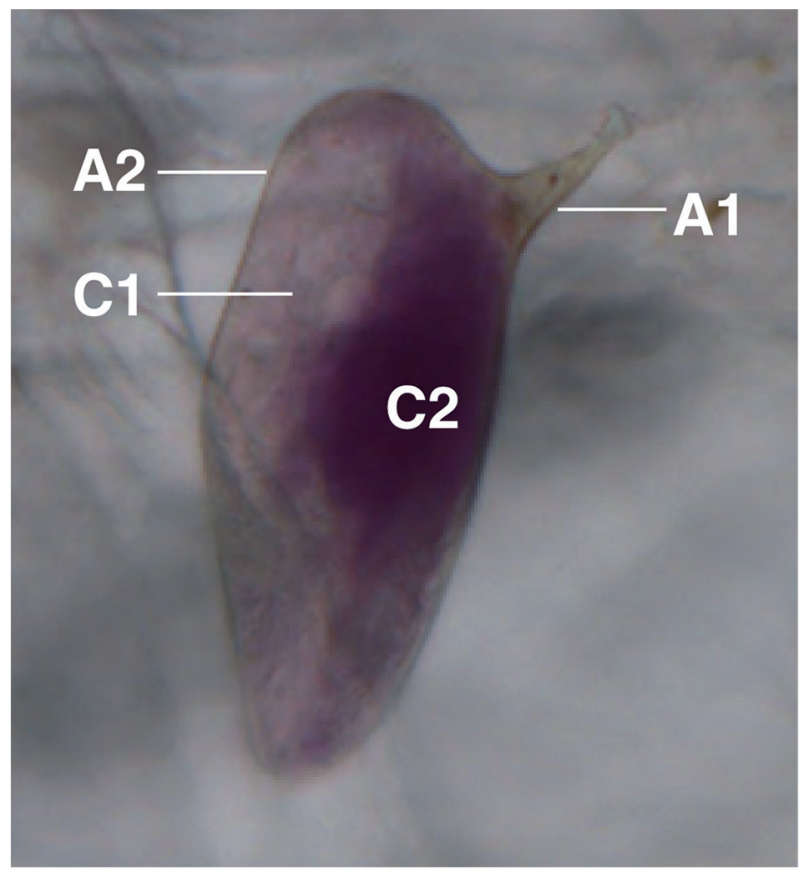

\section{Group A:}

A1: presence of a lateral spine B1: ovoid shape

A2: well-defined shell outline B2: size 150-117 $\mu \mathrm{m} \times 70-40 \mu \mathrm{m}$

\section{Group C:}

\section{C1: space between the shell and the miracidium} C2: purple color

Fig. 1: overview of the six observation criteria used in this study (divided into groups A-C) for the identification of Schistosoma mansoni eggs in sediments prepared according to the Helmintex method and stained with ninhydrin. iability (the lowest distance between lower and higher confidence limits: 0.7 ), the use of this criterion alone, or any other criterion alone, was insufficient for egg identification. Moreover, a clear space between the miracidium and the eggshell (group C criteria) is not always present and is not specific for $S$. mansoni eggs. Therefore, except for the presence of a lateral spine and purple colour, accurate identification using the other four criteria requires more extensive observer training.

In areas with a high prevalence and intensity of infection, recognition of egg characteristics under a microscope is not a serious concern. However, in low endemic areas, more careful training and microscopic examinations are required, particularly where egg detection via microscopy is required as a reference method or cure control. Inter-observer variability may be improved by continuously and rigorously training observers and enhancing quality control measures. However, it was evident that combination 3 (observation of at least one criterion from each group) showed the best performance compared to combinations 1 and 2 . There were significant differences $(\mathrm{p}<0.05)$ between proportional frequencies of all combinations (Fig. 2).

In conclusion, the findings of the current study indicate that identification of S. mansoni eggs was successfully achieved using ninhydrin -stained HTX preparations, when at least one criterion from each group (A-C) was detected. Our findings also indicated that classification as "major/minor" criteria is not recommended. The proposed use of combinatorial observations for identifying S. mansoni eggs was found to be especially useful for egg specimens prepared according to the highly sensitive HTX method. Thus, the high sensitivity of the HTX method, combined with the use of multiple criteria for egg identification, may provide a more reliable diagnosis in settings characterised by extremely low infection intensities.

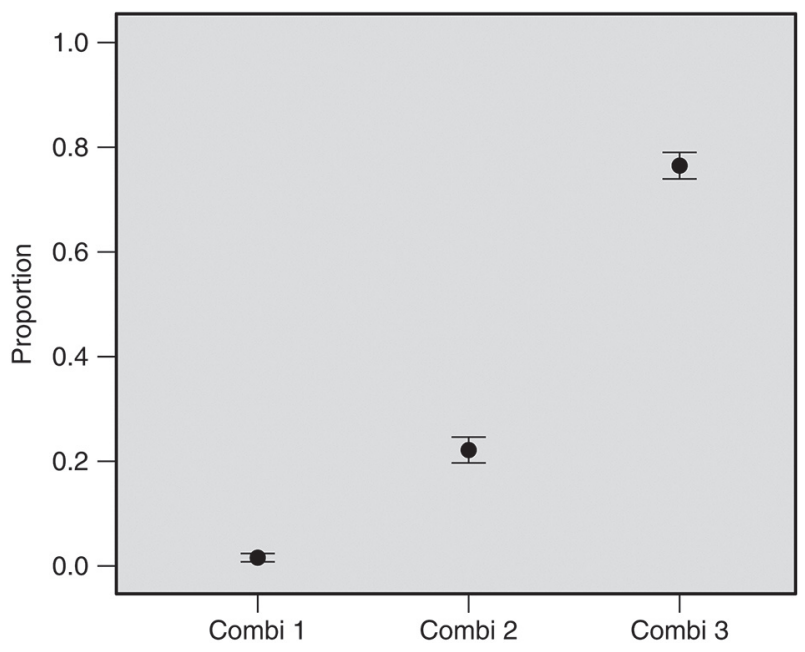

Fig. 2: proportional frequency (95\% confidence intervals) of combinations (Combi) one, two, and three criteria by nine observers. There were significant differences $(\mathrm{p}<0.05)$ between proportional frequencies of all combinations. 
TABLE

Six criteria for identification of Schistosoma mansoni eggs in Helmintex sediments stained by ninhydrin, their frequency, proportional frequency and limits of $95 \%$ confidence intervals (CI)

\begin{tabular}{lccccc}
\hline & & & & \multicolumn{2}{c}{$95 \% \mathrm{CI}$} \\
\cline { 5 - 6 } Criteria & $\mathrm{n}$ & Frequency & Proportion & LL & HL \\
\hline Well-defined shell outline & 900 & 658 & $73,1 \%$ & $70,2 \%$ & $76,0 \%$ \\
\hline Lateral Spine & 900 & 693 & $77,0 \%$ & $74,3 \%$ & $79,7 \%$ \\
\hline Size & 900 & 794 & $88,2 \%$ & $86,1 \%$ & $90,3 \%$ \\
\hline Shape & 900 & 523 & $58,1 \%$ & $54,9 \%$ & $61,3 \%$ \\
\hline Colour & 900 & 897 & $99,7 \%$ & $99,3 \%$ & $100,0 \%$ \\
\hline Space between shell and miracidium & 900 & 683 & $75,9 \%$ & $73,1 \%$ & $78,7 \%$ \\
\hline
\end{tabular}

CI: confidence interval; n: number of observations; LL: CI lower limit; HL: CI higher limit.

\section{AUTHORS' CONTRIBUTION}

RPS, VF, VPF and CGT conceived and designed the experiments; RPS, VF, VPF and CGL performed the experiments; RPS, VF, HRB and CGT analysed the data; RPS, VF, HRB and CGT wrote and reviewed the manuscript. All authors read and contributed to the article.

\section{REFERENCES}

1. Tchuenté LAT, Rollinson D, Stothard JR, Molyneux D. Moving from control to elimination of schistosomiasis in sub-Saharan Africa: time to change and adapt strategies. Infect Dis Poverty. 2017; 6: 42-56. doi: 10.1186/s40249-017-0256-8.

2. Zoni AC, Catalá L, Ault SK. Schistosomiasis prevalence and intensity of infection in Latin America and the Caribbean countries, 1942-2014: a systematic review in the context of a regional elimination goal. PLoS Negl Trop Dis. 2016; 10(3): e0004493. doi. org/10.1371/journal.pntd.0004493.

3. WHO - World Health Organization. Schistosomiasis progress report 2001-2011 and strategic plan 2012-2020. 2013. Available from: http://apps.who.int/iris/handle/10665/78074.

4. De Vlas SJ, Gryseels B. Underestimation of Schistosoma mansoni prevalences. Parasitol Today. 1992; 8(8): 274-7.

5. Kittur N, Castleman JD, Campbell Jr CH, King CH, Colley DG. Comparison of Schistosoma mansoni prevalence and intensity of infection, as determined by the circulating cathodic antigen urine assay or by the Kato-Katz fecal assay: a systematic review. Am J Trop Med Hyg. 2016; 94(3): 605-10.

6. Teixeira CF, Neuhauss E, Ben R, Romanzini J, Graeff-Teixeira C. Detection of Schistosoma mansoni eggs in feces through their interaction with paramagnetic beads in a magnetic field. PLoS Negl Trop Dis. 2007; 1(2): e73.

7. Bell DR. A new method for counting Schistosoma mansoni eggs in faeces. With special reference to therapeutic trials. Bull World Health Org. 1963; 29: 525-30.

8. Favero V, Candido RF, Verissimo CM, Jones M, Saint-Pierre T, Lindholz CG, et al. Optimization of the Helmintex method for schistosomiasis diagnosis. Exp Parasitol. 2017; 177(1): 28-34. doi:10.1016/j.exppara.2017.04.001.

9. Lindholz CG, Favero V, Verissimo CM, Candido RRF, Souza RP, Santos RR, et al. Study of diagnostic accuracy of Helmintex, Kato-Katz, and POC-CCA methods for diagnosing intestinal schistosomiasis in Candeal, a low intensity transmission area in Northeastern Brazil. PLoS Negl Trop Dis. 2018; 12(3): e0006274. http://dx.plos.org/10.1371/journal.pntd.0006274.

10. Lenzi HL, Juberg AD, Coelho PMZ, Lenzi JA. Migração e desenvolvimento de Schistosoma mansoni no hospedeiro definitivo. In: Carvalho OS, Coelho PMZ, Lenzi HL, orgs. Schistosoma mansoni \& esquistossomose: uma visão multidisciplinar. Cap. 3. Rio de Janeiro: Editora Fiocruz; 2008. p. 119. 